\title{
Alpha-particle condensation in nuclei
}

\author{
P. Schuck ${ }^{1}$, H. Horiuchi ${ }^{2}$, G. Röpke ${ }^{3}$, A. Tohsaki ${ }^{4}$ \\ ${ }^{1}$ Institut de Physique Nucléaire, \\ 91406 Orsay Cédex, France \\ 2 Department of Physics, Kyoto University, \\ Kyoto 606-8502, Japan \\ ${ }^{3}$ FB Physik, Universität Rostock \\ -18051 Rostock, Germany \\ ${ }^{4}$ Department of Fine Materials Engineering, \\ Shinshu University Ueda 386-8567, Japan
}

November 2, 2018

\begin{abstract}
A round up of the present status of the conjecture that $n \alpha$ nuclei form an $\alpha$ particle condensate in excited states close to the $n \alpha$ threshold is given. Experiments which could demonstrate the condensate character are proposed. Possible lines of further theoretical developments are discussed.
\end{abstract}

In this article we want to report on the present status of our recent conjecture [1] that at least light $n \alpha$-nuclei may show around the threshold for $n \alpha$ disintegration, bound or resonance states which are of the $\alpha$-particle gas type, i.e. they can be characterised by a self-bound quite dilute gas of almost unperturbed $\alpha$-particles, all in relative s-states with respect to their respective center of mass coordinates and thus forming a Bose condensed state. Such a state is quite analogous to the recently discovered Bose condensates of bosonic atoms formed in magnetic traps. Also there the Bose condensate is characterised by all Bosons occupying the lowest s-wave state of the mean field potential. Of course, in the atomic case the number of atoms can be enormous whereas in the nuclear case we, so far, only consider a handfull of $\alpha$-particles. However, as soon as $n \gg 1$, one can expect condensation characteristics and therefore relatively few $\alpha$ 's may be sufficient. The situation is similar to nuclear pairing where also a very limited number of Cooper pairs are involved and yet most nuclei manifest clear signatures of superfluidity.

Let us shortly repeat what led us to our conjecture. It is well known that the only nucleus which shows a well developed $\alpha$-particle structure in its ground state is ${ }^{8} \mathrm{Be}$. This singular feature was probably not given the attention it desserves. Other nuclei with three, four, ...,n $\alpha$-particles collapse in their groud states to a much denser system where the $\alpha$-particles strongly overlap and probably loose almost totally their identity (see, however discussion below). It seems a very natural 
idea to imagine that, when these $n \alpha$ nuclei are expanded, at some low density individual almost unperturbed $\alpha$-particles reappear forming a weakly self-bound gas-like state with, at most, pairwise two $\alpha$-particle correlations of the ${ }^{8} \mathrm{Be}$-type. Since $\alpha$-particles are Bosons, we conjectured that these low density states form a Bose condesate [1]. At the moment this conjecture is backed by the following findings. Based on the idea of an $\alpha$-particle condensate, we made the following very simple variational ansatz for the $n \alpha$ wavefunction:

$$
<\mathbf{r}_{1} \sigma_{1} \tau_{1}, \ldots \mathbf{r}_{4 n} \sigma_{4 n} \tau_{4 n} \mid \Phi(n \alpha)>=\mathcal{A}\left[e^{-\frac{2}{B^{2}}\left(\mathbf{X}_{1}^{2}+\ldots \mathbf{X}_{n}^{2}\right)} \phi\left(\alpha_{1}\right) \ldots \phi\left(\alpha_{n}\right)\right]
$$

where $\mathbf{X}_{i}=(1 / 4) \sum_{n} \mathbf{r}_{i n}$ is the center of mass coordinate of the i-th $\alpha$-cluster $\alpha_{i}$. The internal wavefunction of the $\alpha$-cluster $\alpha_{i}$ is

$$
\Phi\left(\alpha_{i}\right)=\exp \left[-\left(1 /\left(8 b^{2}\right)\right) \sum_{m>n}^{4}\left(\mathbf{r}_{i m}-\mathbf{r}_{i n}\right)^{2}\right]
$$

The wavefunction of eq. (1) is totally antisymmetrised by the operator $\mathcal{A}$. It is to be noted that the above wavefunction expresses the state where $n \alpha$ clusters occupy the same 0s harmonic oscillator orbit $\exp \left[-\left(2 / B^{2}\right) \mathbf{X}^{2}\right]$ with $B$ an independent width parameter. For example if $B$ is of the size of the whole nucleus whereas $b$ remains more or less at the free $\alpha$-particle value, then the wavefunction (1) describes an $n \alpha$ condensed state for $n \gg 1$. On the contrary, for $B=b(1)$ is a pure Slater determinant. We took the two parameters $B$ and $b$ of (1) as Hill-Wheeler coordinates and calculated for spherical geometry the $0^{+}$spectrum of ${ }^{8} \mathrm{Be},{ }^{12} \mathrm{C}$, ${ }^{16} \mathrm{O}$. Of course, the total center of mass was eliminated. For the effective nucleonnucleon force the one of Tohsaki [2] was adopted who adjusted parameters of a sum of two body and three body Gaussian forces to $\alpha-\alpha$ scattering data and $\alpha$ particle properties long time ago. The Coulomb force was and is, of course, also included. The force, therefore, ressembles an effective force of the Gogny type [3], since the 3-body part gives rise to a density dependence. Thus our theory does not contain any adjustable parameter. The solution of the Hill-Wheeler equation yields one $0^{+}$state for ${ }^{8} \mathrm{Be}$, two for ${ }^{12} \mathrm{C}$, and three for ${ }^{16} \mathrm{O}$. Very close agreement with experiment has been obtained (within some hundred $\mathrm{keV}$ ) for the ground state energy of ${ }^{8} \mathrm{Be}$, for the second $0^{+}$in ${ }^{12} \mathrm{C}$ (with respect to the $3 \alpha$ threshold energy), and for the fifth $0^{+}$in ${ }^{16} \mathrm{O}$ (with respect to the $4 \alpha$ threshold energy). For further details, please consult our papers, either published [1,4] or on the preprint server [5]. The fact that we get correctly, without any adjustable parameters, three states close to the $n \alpha$ desintegration threshold in these nuclei, is very non trivial. For example, it is well known that it is very difficult to obtain the correct position of the second $0^{+}$state in the ${ }^{12} \mathrm{C}[6]$. Only sophisticated resonating group calculations have achieved this [6]. On the other hand, most recent AMD calculations, though clearly showing the $3 \alpha$ structure of the second $0^{+}$state in ${ }^{12} \mathrm{C}$, miss its energy by several $\mathrm{MeV}$ [7]. Concerning the radii, our calculations show that ${ }^{12} \mathrm{C}$ and ${ }^{16} \mathrm{O}$ have, in the threshold states, approximately three times the volume of the one of their ground states. This confirms the dilute gas structure of these states. Furthermore also deformed calculations for ${ }^{8} \mathrm{Be}$ were performed and preliminary results show that the wavefunction (1) allows to reproduce the three lowest members of the rotational spectrum of the ${ }^{8}$ Be very satisfactority [5].

These successes of our theory make us believe that the $\alpha$-condensed states are real and we conjecture that they may exist even for quite high numbers of $\alpha$ - 
particles. For example one may speculate of $10 \alpha$-particles in ${ }^{40} \mathrm{Ca}$. One may also think of a ${ }^{40} \mathrm{Ca}+{ }^{40} \mathrm{Ca}$ head on heavy ion collision. Initially the system gets compressed. If the energy is just right, the decompression may stall approximataly around the $\alpha$-condensate density and the whole system may decay into $\alpha$-particles via the coherent state. Of course, there is temperature and depending on its value more or less $\alpha$ 's may be broken. Such scenarios are nice dreams. But how to prove that these experimental $n \alpha$ threshold states really have Bose condensate character? The difficulty comes from the fact that the condensation occurs in excited states of those nuclei. To prove Cooper pair condensation one makes pair transfer from ground state to ground state and sees that the cross section is enhanced. But how to make an $\alpha$-particle transfer into an $n \alpha$ threshold state? Nevertheless other methods might exist. Imagine that one excites the condensate, for example to a $2^{+}$ state. In ${ }^{12} \mathrm{C}$ such a state exists at $\sim 3 \mathrm{MeV}$ above threshold. The nucleus may then decay into $n=3 \alpha$ 's which can be detected with a multiparticle detector like INDRA. Their measured velocity distribution should be proportional to the Fourier transform of the excited condensate density. The velocity distribution will therefore be rather narrow, if it corresponds to a wide density distribution, as assumed for the condensate. In heavy ion reactions rotational states are excited. A rotating condensate should have a strongly reduced moment of inertia with respect to the rigid body value [8]. Other experiments may be imagined to put the condensate character of the $n \alpha$ threshold states into evidence. An indirect indication that the $\mathrm{O}_{2}{ }^{+}$-state in ${ }^{12} \mathrm{C}$ has extended character may come from the recent measurement of the spin-orbit splitting of of the extra neutron in ${ }^{13} \mathrm{C}$ when added to this state (v. Oertzen et al., this meeting). Indeed the spin-orbit splitting in ${ }^{13} \mathrm{C}$ corresponding to the $0_{2}^{+}$-state in ${ }^{12} \mathrm{C}$ is only half of the one of that corresponding to the $0_{1}^{+}$-state. A wider and flatter single particle potential in this excited state with respect to the one for ground state could possibly explain this fact.

Adding neutrons to the $\alpha$-condensate is another very interesting subject. Adding neutrons to ordinary Cooper-pair condensates quickly destroys superfluidity due to the blocking effect [8]. This also holds true for neutron-proton Cooper pairing [9]. However, adding neutrons to a low density Bose condensed gas of deuterons does not affect the deuteron condensate because the Pauli principle is not effective [9]. With the $\alpha$-particle condensate we may well have an analogue to the latter situation of the deuterons. Even on the contrary, some neutron excess may help to stabilise the condensate (v. Oertzen, this meeting) making the question of the upper limit of the number of condensed $\alpha$ 's an even more intriguing one. Remember that ${ }^{8} \mathrm{Be}$ is unbound whereas ${ }^{9} \mathrm{Be}$ is bound!

Let us now come to the question to what extend even the ground state of $n \alpha$-nuclei may show superfluid characteristics. The contour plots $E(B, b)=<$ $\Phi_{n \alpha}|H| \Phi_{n \alpha}>/<\Phi_{n \alpha} \mid \Phi_{n \alpha}>$ show [1] that the absolute minima of the energy surfaces have an energy gain of several Mev with respect to the HF-limit $B=b$. However, one must be careful with the conclusion about this fact. Let us discuss the issue with the more familiar situation of standard Cooper pairing. In nuclear physics we are used to call a nucleus superfluid, when the BCS equations have a non-trivial solution with a finite gap (contrary to infinite matter where a BCSsolution always exists for arbitrary weak attractive interaction, in finite systems the BCS equations not always have a solution because of finite level spacings and shell fluctuations of the single particle level density). As a rule, therefore, nuclei at or near shell closure show no pair coherence whereas open shell nuclei are superfluid. The BCS wavefunction $\left|B C S>=\exp \left(P^{+}\right)\right| v a c>$, with $P^{+}=\sum_{k} z_{k} a_{k}^{+} a_{\bar{k}}^{+}$ 
the Cooper pair creation operator, does not conserve particle number. A better variational wavefunction is therefore the so-called number projected BCS wavefunction $\left|P B C S>=\left(P^{+}\right)^{N / 2}\right| v a c>$ where $\mathrm{N}$ is, e.g. the number of neutrons. Performing a minimisation of the energy with respect to the parameters $z_{k}$ with $\mid P B C S>$, we will find that a non-trivial solution always exists, i.e. even doubly magic nuclei will experience a gain in energy with respect to the HF-approach. Nontheless we will not consider magic nuclei as superfluid. This illustrates that $\mid P B C S>$ theory allows to treat two body correlations in non superfluid nuclei as well as it contains the superfluid case. We recognise that our $\alpha$-condensed wavefunction (1) is analogous to number projected BCS and therefore may describe situations with four particle correlations as well as situations with $\alpha$-particle condensation. We believe that (1) largely describes $\alpha$-like correlation in the ground states of $n \alpha$ nuclei, whereas the threshold states can be considered as condensates. This statement is corroborated by our recent investigations of $\alpha$-particle consensation in infinite nuclear matter [10]. There it turns out that $\alpha$-particle consensation ceases to exist once the density reaches values where the $\alpha$ 's start to overlap substantially. However, in finite nuclei this needs more carefull investigation because $\alpha$ 's on the surface of, say, ${ }^{40} \mathrm{Ca}$ in its ground state, may still experience low density whereas in the core the $\alpha$ 's are strongly overlapping. In this respect, an intriguing fact of a pronounced even-odd effect in the number of deuterons has recently been revealed by K.H. Schmidt et al [11] (see also this meeting). Indeed light nuclei with an even number of "deuterons" ( $n \alpha$ nuclei) have systematically a lower binding energy than those with an odd number of "deuterons". This rather pronounced "even-odd" effect hints to pairing of "deuterons". As a matter of fact sometime ago we have already attempted to describe $\alpha$-particle condensation within a Boson picture of nucleon pairs in applying a bosonic BCS approach to the IBMII model [12]. However, still further investigations must be performed before drawing any definite conclusions. For example, are $\alpha$-particle transfer reactions enhanced in $n \alpha$ nuclei? Does the band of ground states energies of neighbouring n $\alpha$ nuclei show rotational or vibrational character? This in analogy to pair vibrations and pair rotations, well known from the physics of ordinary pairing [8]. Another item could be whether the rotational ground state band of, e.g. ${ }^{24} \mathrm{Mg}$ shows a rigid or a superfluid moment of inertia.

Let us also make some further remarks about the fact that our wavefunction (1) yields an energy minimum several MeV lower than the HF limit [1]. One may ask the question, whether present Skyrme- or Gogny-type of HF-calculations would also get a lowering of several $\mathrm{MeV}$ when $\alpha$-type of correlations were included. We think that the answer is 'yes' but then the parameters of the forces would have to be readjusted. On the other hand, the force of Tohsaki [2] used here has been adjusted with correlations included. It therefore is not astonishing that our energy minima lie close to experiment and the HF-limit higher.

Further light on these questions could be shed in formulating a theory of $\alpha$ particle condensation with a coherent, $\alpha$-particle non conserving, state $\left|\Phi_{\alpha}\right\rangle$, rather than with our number projected state $\mid \Phi_{n \alpha}>$ of eq. (1). For this, we have to introduce a Bogoliubov transformation among Fermion pairs, rather than of single Fermions like in the case of Cooper pairing. This can be done in introducing quasi-pair operators $Q_{\nu}^{+}=\sum_{k, k^{\prime}}\left[X_{k, k^{\prime}}^{\nu} a_{k}^{+} a_{k^{\prime}}^{+}-Y_{k, k^{\prime}}^{\nu} a_{k^{\prime}} a_{k}\right]$ and demanding the existence of a coherent state by $Q_{\nu} \mid \Phi_{\alpha}>=0$. Minimising the average energy 


$$
S_{1}=<\Phi_{\alpha}\left|\left[Q_{\nu}\left[H^{\prime}, Q_{\nu}^{+}\right]\right]\right| \Phi_{\alpha}>/<\Phi_{\alpha}\left|\left[Q_{\nu}, Q_{\nu}^{+}\right]\right| \Phi_{\alpha}>
$$

with respect to the $X, Y$ amplitudes leads to the eigenvalue problem

$$
<\left[\delta Q,\left[H^{\prime}, Q_{\nu}^{+}\right]\right]>=E<\left[\delta Q, Q_{\nu}^{+}\right]>
$$

with $H^{\prime}=H-\mu N$. For a two body Hamiltonian the double commutator contains one and two body densities. Since the X,Y-amplitudes form a orthonormal set, the relation $Q^{+}=X a^{+} a^{+}-Y a a$ can be inverted and then the two body densities can be expressed in terms of $\mathrm{X}, \mathrm{Y}$ and one body densities. The latter can be calculated from a single particle Green's function consistent with the whole scheme. This leads to an $\alpha$-particle (four-body) gap equation and an $\alpha$-particle order parameter $\kappa_{1234}=<\Phi_{\alpha}\left|a_{1} a_{2} a_{3} a_{4}\right| \Phi_{\alpha}>$ in analogy to pairing of single Fermions. To good approximation the vacuum is given by

$$
\left|\Phi_{\alpha}>\sim \exp \left[\sum z_{1234} a_{1}^{+} a_{2}^{+} a_{3}^{+} a_{4}^{+}\right]\right| v a c>
$$

with $z_{1234}=\left(Y X^{-1}\right)_{1234}$. Partly, this formalism, called Self Consistent Quasiparticle RPA (SCQRPA) has been worked out in [13]. In general, the numerical solution of the corresponding equations is very involved. However, approximating the wavefunctions by parametrised Gaussians like in (1), such calculations may seem feasable. Indeed $z_{1234}$ corresponds to a single $\alpha$-particle wavefunction in (1):

$$
z \sim \kappa \sim \exp \left[-\frac{2}{B^{2}} \mathbf{X}^{2}\right] \Phi(\alpha)
$$

The problem will be to obtain from this ansatz for $z$ the corresponding expressions for $X$ and $Y$. This would allow to treat practically any number of $\alpha$-particles, whereas the present number projected ansatz (1) is limited to a rather low value of $n$ because the explicit antisymmetrisation quickly increases in complexity. Another way to treat large numbers of $\alpha$ 's is to develope an effective theory, considering the $\alpha$ 's as elementary particles, i.e. structureless bosons. Equations of the Gross-Pitaevskii-type or corresponding Hill-Wheeler equations may then be developed. The possibility to treat large numbers of $\alpha$-particles is important in order to decide where are the limits of a self-bound gas of $\alpha$-particles.

A further speculation related to our studies above is that there may also exist condensates of heavior clusters, particularly of ${ }^{16} \mathrm{O}$ nuclei. For example, ${ }^{48} \mathrm{Cr}$ might possess an excited $0^{+}$state close to the three ${ }^{16} \mathrm{O}$ threshold analogous to the $0_{2}^{+}$in ${ }^{12} \mathrm{C}$, or ${ }^{64} \mathrm{Gd}$ may show a four ${ }^{16} \mathrm{O}$ gas like state. Probably also partial Bose condensates exist. For example exciting ${ }^{32} \mathrm{~S}$ a state with a ${ }^{16} \mathrm{O}$ - core +4 loosely bound $\alpha$-particle may appear. Such states are being analyzed by M. Brenner et al. [14]. This concludes the round up of the present status of $\alpha$-particle condensation in nuclei. We indicated present theoretical results hinting to the existence of such states in $n \alpha$ nuclei. We discussed consequences of such states, open problems, and perspectives.

\section{Acknowledgements}

We thank Y.Funaki and T.Yamada for fruitful discussions and contributions to this work. 


\section{References}

[1] A. Tohsaki, H. Horiuchi, P. Schuck, G. Röpke, Phys. Rev. Lett. 87 (2001) 192501-1

[2] A. Tohsaki, Phys. Rev. C 49 (1994) 1814

[3] J. Decharge, D. Gogny, Phys. Rev. C 21 (1980) 1568

[4] P. Schuck, A. Tohsaki, H. Horiuchi, G. Röpke, The Nuclear Many-Body Problem 2001, 271-278, W. Nazarewicz, D. Vretenar (eds.), 2002 Kluwer Academic Publishers

[5] Y.Funaki, H. Horiuchi, A. Tohsaki, P.Schuck, G. Röpke, nucl-th/0207022

[6] Y. Fujiwara, H. Horiuchi, K. Ikeda, M. Kamimura, K. Kato, Y. Suzuki, E. Uegaki, Prog. Theor. Phys. suppl. No 68 (1980) 29

[7] H. Horiuchi, private communication

[8] P. Ring, P. Schuck, The Nuclear Many Body Problem, Springer 1980

[9] U. Lombardo, P. Nozières, P. Schuck, H.J. Schulze, A. Sedrakian, Phys. Rev. C 64 (2001) 064314

[10] G. Röpke, A. Schnell, P. Schuck, P. Nozières, Phys. Rev. Lett. 80 (1998) 3177

[11] J.J. Gaimard, K.H. Schmidt, Nucl. Phys. A 531 (1991) 709

[12] Y.K. Gambhir, P. Ring, P. Schuck, Phys. Rev. Lett. 51 (1983) 1235

[13] J. Dukelsky, P. Schuck, Phys. Lett. B 387 (1996) 233

[14] V.V. Lazarev, S.E. Belov, M. Brenner, K.A. Gridnev, T. Kurtukian Nieto, D.D. Caussyn, N.R. Fletcher, J.A. Liendo, S.H. Myers, Phys. of Atomic Nuclei (2000), Proceedings of the International Conference "Clustering Phenomena in Nuclear Physics", St Petersburg 